\title{
On the Integration of the UMTS and B-ISDN system
}

\author{
G. Karagiannis, B.J.F. van Beijnum, I.G.M.M. Niemegeers \\ Centre for Telematics and Information Technology (CTIT) \\ University of Twente, P.O.Box 217, Enschede, the Netherlands \\ Phone: +31 534893747 ; Fax: +31 53489 3247; \\ Email:karagian@ctit.utwente.nl
}

\begin{abstract}
The main theme of this paper is the presentation of some critical issues such as transcoding, transport interworking, handover, congestion control and network scalability, that influence the integration of the UMTS and B-ISDN. Solutions to these issues will also be discussed. Furthermore, we will address a number of quantitative (i.e. performance) aspects of an integrated UMTS/B-ISDN system.
\end{abstract}

\section{Keywords}

Mobile communication, B-ISDN, intelligent networks, interworking, transcoding, handover, network scalability, congestion control, performance analysis.

\section{INTRODUCTION}

The use of mobile communications has increased rapidly over the last fifteen years and a similar growth is being expected for the next ten years. In the early 80 s the first generation analogue cellular phones were introduced and the main focus was put on the business sector. The second generation of mobile telecommunication systems has recently been introduced. It is expected that this second generation will be adequate to fulfil the needs until the beginning of the next century. It has been predicted that by the year 2000 the European market will have over one hundred million mobile terminals. By that time, there will be a need for a new generation of mobile telecommunication systems.

To fulfil these future needs, the MONET project (MObile NETworks) addressed the development of a third generation mobile system which is known as Universal Mobile Telecommunication System (UMTS). The MONET project was sponsored by the European Committee as part of the RACE-II programme. 
One of the main objectives of the MONET project was to develop a system in which UMTS and B-ISDN are integrated so that broadband mobile services can be provisioned.

In order to allow such an integration of UMTS and B-ISDN a number of critical technical problems had to be solved. This paper gives an overview of these problems and discusses possible solutions for them.

This paper is organised as follows. In Section 2 we give an overview of the UMTS/B-ISDN architecture. Typical problems related to mobile communication are: transport interworking, transcoding and handover. They are discussed in Sections 3. Two more issues are addressed in Section 4 and 5: scalability and congestion control. Some first experimental performance analysis results are presented Section 6. In Section 7 conclusions are presented.

\section{UMTS/B-ISDN ARCHITECTURE}

The design and standardisation of the integrated UMTS/B-ISDN system takes place in a stepwise fashion. It starts with user and functional requirements and finally results in an overall architecture: a blueprint of possible realisations of the integrated UMTS/B-ISDN system (MONET, 1995c). The design trajectory followed for UMTS/B-ISDN integration is based on the ITU-T 'stage method' (ITU-T, I.130). An important aspect in the design of the UMTS/B-ISDN architecture is the specification of mobility procedures and their mapping onto system blocks and interfaces.

Each procedure is specified by a single functional model (FM). A functional model itself, may be specified in terms of several functional entities and their interactions. These interactions between functional entities are specified in terms of information flows (IFs).

In the MONET project, UMTS has been specified as well as the integration of UMTS and a B-ISDN backbone network. As far as mobile communication is concerned, MONET uses concepts encountered in intelligent networks (IN), for details we refer to (Katoen, 1995), (Broek, 1995), (Karagiannis, 1996) and (MONET, 1995c). In our study we go one step further and consider a backbone network that itself is an integration of B-ISDN and Intelligent Network (IN), the European ACTS project INSIGNIA (Integration of IN and B-ISDN on ATM platforms) is specifying and implementing such an integration (INSIGNIA, 1996).

Figure 1 illustrates the interconnection of the basic physical entities in a UMTS/B-ISDN architecture. Starting at the user side, there are fixed terminals (FT) and mobile terminals (MT). A fixed terminal is directly connected to the backbone B-ISDN network. The backbone network itself may be an integration of B-ISDN and an intelligent network (IN). 


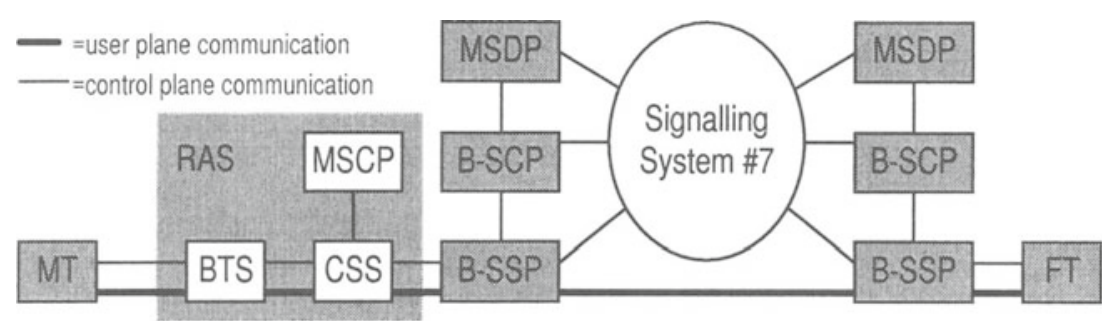

Figure 1 Overview of the integrated UMTS/B-ISDN architecture.

In this case, the backbone network would include physical entities such as: broadband service switching point (B-SSP) that implements UNI/NNI switching and IN interaction; broadband service control point (B-SCP) that implements the service control logic to provide intelligent network services (this includes mobility specific control logic); mobile service control point (MSCP) that implements mobility specific service control logic (such as handover); and mobility service data point (MSDP) that implements a set of functions for mobility specific service data (for instance, mobile terminal location).

A mobile terminal has access to the backbone B-ISDN network via a Radio Access System (RAS), in which the Base Transceiver Station (BTS) manages radio access and the Cell Site Switch (CSS) takes care of call control and it controls the connection to the backbone network.

For the communication between the physical entities in the integrated UMTS/B-ISDN network, a set of protocol stacks have been defined. As far as NNI is concerned we refer to (Colombo, 1994). Our focus will be on the UNI. Figure 2 shows the protocol stacks that are involved in the user plane communication between a mobile terminal and a fixed terminal. UNI radio access incorporates the protocol layers: Radio Physical layer (RPHY), Radio Data Link layer (RDL) and UMTS ATM Adaptation layer (UAL). UNI fixed access incorporated the protocol layers: Physical layer (PHY), ATM and ATM Adaptation Layer (AAL). At application level we have the service application layer (APPL).

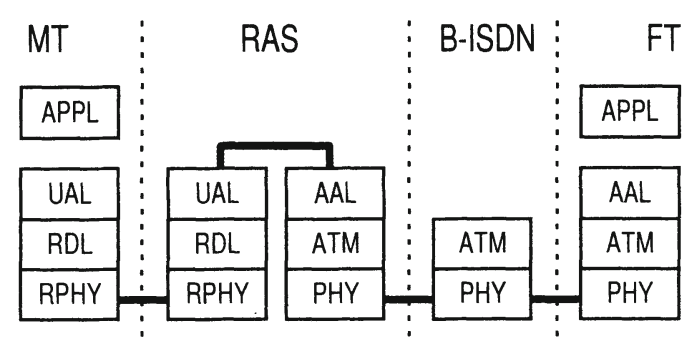

Figure 2 Protocol stacks for user plane communication. 


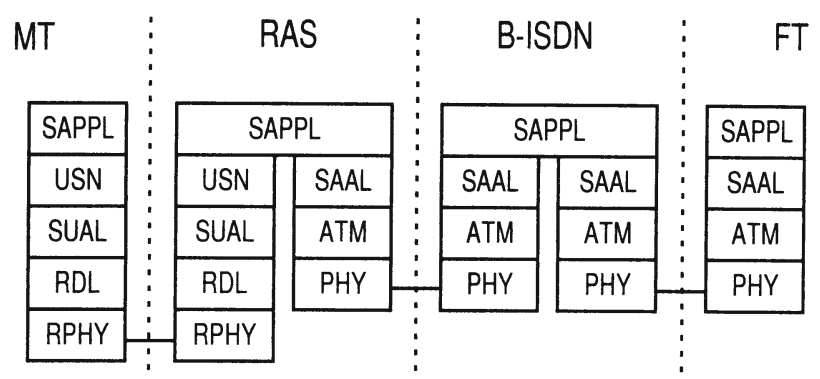

Figure 3 Protocol stacks for control plane communication.

UNI control (or signalling) plane communication is established as shown in Figure 3. Radio access incorporates the layers: Signalling UMTS ATM Adaptation layer (SUAL), UMTS Signalling Network layer (USN) and Signalling Application layer (SAPPL). The UMTS Network layer handles all non-call related mobility functionality such as: paging, location updates (Norp, 1994). Call related signalling is handled by the signalling application (SAPPL). The specification of the Signalling Application layer is one of the results of the MONET project (MONET, 1995c). The fixed part of the UNI control plane needs enhancements of existing UNI signalling in order to support call related mobility functions.

\section{INTEGRATION OF UMTS AND B-ISDN}

In this section the three major problems encountered in integrating UMTS and BISDN, namely transport interworking, transcoding and handover, are discussed.

\subsection{Transport interworking}

The different UMTS and B-ISDN protocol stacks require interworking functions that extract information from one protocol stack and reformat it for onward transmission via a different protocol stack. Interworking might be defined at different levels in the protocol stack.

In the MONET project three different options for interworking have been identified, for a detailed discussion we refer to (MONET, 1995a), (Marchent, 1996). We give a short explanation of the options and use Figure 2 as reference configuration:

- Interworking at source (application) level: This interworking type is service dependent (Immonen, 1995), detailed knowledge and requirements of the data source must be known to control any interworking. The UAL (in the RAS) must interwork with a suitable AAL (in the RAS), this AAL corresponds to the AAL used by the other end user (FT). 
- Interworking at AAL level: This interworking type is service independent, i.e. it does not need source information to control the interworking. AAL packets are transported transparently to the mobile terminal (MT). The interworking could be achieved in the following way. The MT service application (APPL) encapsulates AAL packets in UAL packets, and the FT service application (APPL) encapsulates UAL packets in AAL packets. At the RAS the interworking from the MT to the FT is performed by extracting the AAL packets from the UAL packets. In the opposite direction the RAS provides interworking by extracting the UAL packets from the AAL packets. The transport interworking function is provided by using the information carried by the AAL headers.

- Interworking at ATM level: This interworking type is also service independent (see also (Immonen, 1995)), where ATM cells are transported transparently through the air interface. The RAS provides interworking between the UAL and the ATM layer. Therefore, the AAL layer is not used in the RAS. The interworking could be achieved in the following way. The MT APPL first encapsulates AAL packets into ATM cells, and subsequently the ATM cells are encapsulated in UAL packets. The FT APPL encapsulates UAL packets into AAL packets. At the RAS the interworking from the MT to the FT is performed by extracting the ATM cells from the UAL packets. In the opposite direction the RAS provides interworking by extracting first the AAL packets from the ATM cells and subsequently by extracting the UAL packets. The ATM cell header is used to control the transport interworking function.

Which of the three interworking options can be applied best is still subject to further research, performance evaluation could be one of the important tools to guide this design decision.

\subsection{Transcoding}

Transcoding is an interworking function that changes the source coding format of the user information transported through several links during an end-to-end connection.

In present-networks such as N-ISDN and GSM (Global System Mobile) transcoding only occurs on network boundaries, because within one network exactly one coding scheme is used for a specific service (i.e. telephony). The advantage of this topology is that the network knows whether transcoding is required before the call is routed to the destination terminal. In this way the network is able to select a route in which a transcoder is permanent physically connected, such that no additional switching capacity is required and no call setup delay occurs.

With the development of multimedia applications an increase of the number of audio and video coding schemes is foreseen. Therefore, in the future integrated UMTS and B-ISDN system a generic control function for transcoders can be 
foreseen that is able to insert transcoders where necessary, in a fashion transparent to the user. The transcoding function is in this case implemented as a Specialised Resource Function (ITU-T, Q.1214).

A disadvantage of such a transcoding scheme, however, is an increased overhead in the network for establishing calls and, correspondingly, an increased call set-up delay time. Therefore, for the most commonly used services there will continue to be a strong need for standardising the coding formats.

It is foreseen that in the integrated UMTS and B-ISDN system the speech transcoding will be the most applied transcoding type. Therefore it could be investigated whether a dedicated "telephony with compressed voice" service can be introduced, see also (ATM Forum, 1994). The main transcoding functions that will be needed for this service type relate to the speech transcoding between 'compressed voice' and the standard PCM (Pulse Code Modulation) speech coding format as used in N-ISDN.

In the MONET project (MONET, 1995b) an implementation of the 'compressed voice telephony' service is presented. A terminal (mobile or fixed) indicates the compressed voice telephony service and a corresponding cell rate.

If the call is routed to a gateway (similar to the N-ISDN gateway), the gateway applies speech transcoding to $64 \mathrm{kbps}$ PCM and uses signalling messages to the B-ISDN according to the normal narrowband telephony service.

If the call is routed towards a terminal (mobile or fixed), the destination exchange allows the destination terminal to negotiate the type of speech coding (to allow interworking with terminals that do not support compressed voice). To this end the destination exchange adds an information element for $64 \mathrm{kbps}$ PCM encoding to the set-up message and also the bandwidth is negotiated (ITU-T, Q.2962) with a normal peak cell rate of $64 \mathrm{kbps}$ and an alternative peak cell rate according to the compressed voice encoding scheme. If the destination terminal supports the compressed voice service it will select this and no interworking is required. If the destination terminal selects the PCM coding, the destination exchange has to insert a transcoder at connect time.

Further investigations could be accomplished to identify efficient algorithms for the control of the transcoding functions, i.e. identification of the coding types used at the source and destination and of the location of the transcoding function.

\subsection{Handover}

During a call, handover $(\mathrm{HO})$ is required to transfer the MT connection to each new BTS when the MT roams. From the user's point of view, handover should be seamless. This implies that a handover should not degrade the QoS (Quality of Service) for the connection as perceived by the user, for the support of UMTS/BISDN services, such as Broadband Video on Demand and Broadband Video Conference (INSIGNIA, 1996). 
In (Karagiannis, 1997) two different HO mechanisms that affect both user and signalling part are described. The first mechanism is the HO synchronised switching mechanism and the second is the $\mathrm{HO}$ with multicast support mechanism. The first handover mechanism is relatively easy and it does not use any difficult ATM multicasting nor resynchronisation in the network. The scenario assumes that there is sufficient time available where all the data and history information of the old path can be transferred to the MT before the actual handover to the new path takes place. This means that time is available to empty the buffers on the old path and to stop the interleaving and transcoding process on the old path. It is possible that this time is not available. This implies that the time between a handover decision and the actual handover is too short to end the transmission on the old path gracefully (e.g., ending the interleaving matrix, ending transcoder functions, emptying intermediate buffers). A possible solution for this problem is given by the second $\mathrm{HO}$ mechanism where in the downstream direction multicast connections are used in the core network. By using multicast connections to all possible new target RAS systems, the history can already be built up on the possible target paths. At the time the handover needs to take place the history on the old path can be ignored, and the history at the new path can be used to resume transmission. The HO mechanisms require use of end delimiters. When information is switched between old and new paths (both upstream and downstream), the last information on the old path is marked with an end delimiter. The destination will receive information from the old path until this end delimiter and then switch to receiving information from the new path. The end delimiter might be implemented in the ATM header or as an Operations and Maintenance ATM cell.

\section{NETWORK SCALABILITY}

For any innovative communication system to be commercially interesting it is of crucial importance to be 'scalable'. Network scalability refers to the ability to increase the 'size' of the network while maintaining Quality of Service and network performance criteria. An initial list of distinct, though not necessarily independent, parameters for network size are:

- number of nodes and links in the network;

- number of users connected to the network;

- geographic spread covered by the network, and

- number and type of services provided by the network.

We shall name these parameters the scalability parameters. When increasing the size of a network, we want to meet certain network performance criteria. To evaluate the quantitative aspects of a network, hence its performance, we use network performance measures. According to (ITU-T, I.350) three main performance criteria can be distinguished, these are: speed, accuracy and 
dependability. Specific performance measures related to these criteria are: throughput, error probability and blocking probability.

Basically, the investigation of network scalability is an analysis or evaluation of the network performance measure as function of the scalability parameters. This concept can be brought one step further in which we take so called resource parameters into consideration as being our instruments to change and improve the network performance. Within the context of the UMTS/B-ISDN integration a small list of such network resource parameters is shown in Table 1. Based on these three notions (scalability parameters, performance measures and resource parameters) we can construct an iterative procedure for a scalability experiment. It comprises the following steps:

Step 0: - Select network performance measure(s) of interest.

- Select the scalability parameter(s) of interest and identify their value domain.

- Identify the desired relationship of the performance measure as function of the scalability parameter.

Step 1: - Determine the performance measure as function of the scalability parameter (e.g. through discrete event simulation).

Step 2: - If the result satisfies the desired relationship then the scalability experiment halts, else the cause(s) of the scalability problem should be identified.

Step 3: - Express the problem in terms of one or more changes in the resource parameters, apply those changes to the network (model).

- Return to Step 1.

In Figure 4 a scalability experiment is illustrated. When starting such an experiment, we first define the performance measures of interest (for instance throughput), the scalability parameters that we want to consider (for instance number of users) and finally we define the desired value of the performance measure as function of the scalability parameter (assume this is curve $Y_{0}$ as shown in Figure 4). In the next step we determine, given the network model, the actual value of the performance measure as function of the scalability parameter resulting in graph $\mathrm{Y}_{1}$. Assume that we have identified the scalability problem and that we have found a way to solve the problem, in the next iteration simulation results produces curve $Y_{2}$. By further iteration steps we obtain curve $Y_{3}$, which satisfies the desired performance as function of the scalability parameter. We can therefore halt the scalability experiment. 
Table 1 Examples of UMTS/B-ISDN resource parameters

\begin{tabular}{|c|c|c|c|}
\hline resource parameter & physical entity & explanation & $\begin{array}{l}\text { affected performance } \\
\text { measure }\end{array}$ \\
\hline processing speed & B-SCP, B-SSP & $\begin{array}{l}\text { speed at which a message } \\
\text { can be processed }\end{array}$ & delay \\
\hline routing algorithm & B-SSP & $\begin{array}{l}\text { the selected path along which } \\
\text { connections are set up }\end{array}$ & blocking probability \\
\hline scheduling & $\begin{array}{l}\text { RAS, B-SSP, } \\
\text { B-SCP }\end{array}$ & $\begin{array}{l}\text { the order in which incoming } \\
\text { messages are being } \\
\text { processed }\end{array}$ & $\begin{array}{l}\text { delay, blocking } \\
\text { probability }\end{array}$ \\
\hline $\begin{array}{l}\text { service load } \\
\text { sharing }\end{array}$ & B-SCPS & $\begin{array}{l}\text { distribution of IN service } \\
\text { control over several B-SCPS }\end{array}$ & $\begin{array}{l}\text { delay, blocking } \\
\text { probability }\end{array}$ \\
\hline
\end{tabular}

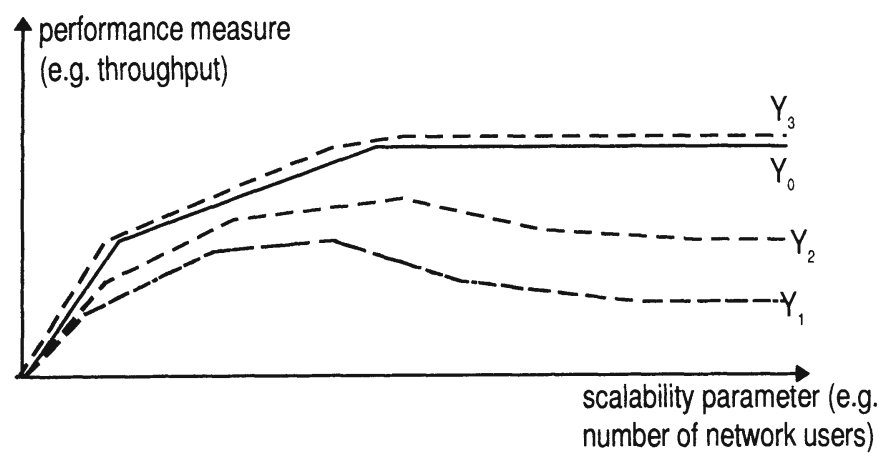

Figure 4 Illustration of a scalability experiment.

\section{CONGESTION CONTROL}

When the integrated UMTS/B-ISDN architecture has to process an incoming load larger than its capacity, the performance (for example throughput) of the system degrades and eventually the users will experience long delays or a high percentage of service refusals (blocked calls). This state of the system is called congestion. Congestion problems and associated performance degradation can be solved by using appropriate congestion control mechanisms. These mechanisms can be characterised as combinations of admission and flow control algorithms, see also (Schwarz, 1995).

The admission control mechanisms will impose to a receiving node, e.g. MT, to admit a call only if sufficient end-to-end bandwidth is available, i.e. from the calling party to the called party. But in the integrated UMTS and B-ISDN system a mobile terminal can move from a good QoS radio cell to a poor QoS radio cell 
and the network node (B-SSP) that is involved in the provisioning of the service must adjust its transmission rate accordingly. This adjustment will create significant problems in the admission control for multimedia applications.

The flow control mechanism is mainly information rate control. The mechanism is activated by a receiving node, e.g. CSS, and throttles the rate at which information is send by its source, e.g. B-SSP. The flow control mechanism that may be used in the integrated UMTS and B-ISDN system differs from the one used in current fixed networks in the following:

- The variation of the transmission medium characteristics of wireless links (in different radio cells, or even within one radio cell).

- Handling of multiple receivers (mobile and fixed) connected in multiparty call associations.

Because of these differences, it is expected that flow control may be activated on traffic sources more often by mobile receivers (scarce radio resources) than by fixed receivers. An other problem occurs when conflicting flow control requests are issued by several receivers, which may either be fixed or mobile, that are involved in the same call association.

A possible new problem that occurs is due to mobility features, such as handover, which may have a considerable effect on the B-SCP processing. In case the B-SCP has to support both MT and FT in a service session, it is likely that the B-SCP will reach a congestion state sooner, when compared to a situation in which only FT are involved in a service session. When congestion in the BSCP occurs, and thus the congestion control mechanism is active, the mechanism has to ensure that each source (relative to the $\mathrm{B}-\mathrm{SCP}$ ) gets a, predefined, fair share of the B-SCP processing capacity.

In the literature, we refer to (Smith, 1995), (Pham, 1994), (Bellcore, 1993) and (Turner, 1986), different congestion control mechanisms for fixed systems are described. These could be used as a starting point for the development of new congestion control mechanisms to solve the congestion problems mentioned earlier. In the following, we briefly describe a set of criteria that might be used to compare congestion control mechanisms.

The effectiveness of a congestion control mechanism depends heavily on the system design and on the traffic situation. An effective and efficient method of how a system can deal with moderate traffic overload situation is to adapt system and, only under more extreme traffic overload conditions, to adjust traffic sources to system capacity (Houck, 1994). The main objectives of congestion control can be summarised as follows:

- Provide good throughput even in extreme overload situations.

- The message delays under overload situations should not be much larger than those in normal situations.

- Provide high reliability. Even under high overload situations the system should not break down. 
- Ensure system fault tolerance and responsiveness.

- In the situation when service degradation occurs, ensure that this is accomplished gracefully.

- Ensure that under overload situations the system capacity is allocated to the system users on a fair predefined manner.

Here we describe some of the important criteria that can be used to analyse congestion control mechanisms, see also (Smith, 1995), (Korner, 1994), (Hubig, 1994) and (Houck, 1994).

1. Robustness: A congestion control mechanism should ensure that the controlled entity (B-SSP or B-SCP) will provide a near maximum throughput even in extreme overload situations. ITU has recommended in (ITU-T, Q.543) that with an overload of $50 \%$ above the engineered capacity, the throughput must not fall below $90 \%$ of the engineered capacity. The ITU-T recommended throughput curve criterion is a robustness criterion.

2. Overhead: The amount of processing capacity needed by the congestion control algorithm should be small relative to the real-time spent for processing input traffic.

3. Traffic input message delays: The traffic input message delays during overload should not be much larger than those under normal conditions.

4. Responsiveness: This is a superposition of the following times:

- detection: the time between the onset of a congestion event in the system until the time that the control mechanism enters a state denoting that the system is in congestion;

- reaction: The time between the detection of a congestion situation in the system until the time that the control mechanism takes an action to attempt to relieve the congestion;

- operation: The time between the initiation of an action on the part of the congestion mechanism in order to relieve the event and the time that the system resumes normal operation.

5. Control of the non-essential processing activities during overload: The nonessential processing activities such as administrative and/or maintenance activities can be suspended during overload situations.

6. Control of the processing activities that ensure system fault tolerance: The congestion control mechanism should ensure that the system fault tolerance, e.g., detection and recovery from data errors, under overload situations should not be adversely affected.

7. Oscillations of congestion states due to statistical fluctuations of traffic: The congestion control mechanism should tolerate (not be activated during) normal traffic fluctuations. This criterion can be applied to analyse the congestion detection method used in the congestion control mechanism.

8. Throughput degradation in adjacent nodes: The congestion control mechanism used in a congested part of a network should not slow or stop the processing activities in a way that would create congestion in adjacent nodes. 
9. Fairness: A congestion control mechanism is fair if the system capacity (BSSP or B-SCP node capacity) is allocated to the system users in accordance with some predetermined definition of equity. An effective fairness criterion is quite difficult to specify. Some important factors that can be used to specify the fairness criterion are the following:

- the rate of reattempts of the rejected system users. This rate should be kept relatively low;

- the services used. System users that are using business services (e.g. Video Conference) may require and may obtain more system capacity than users that are requiring domestic services (e.g. Video on Demand);

- the time of the day that a specific service is used. For example a VoD service system user may obtain in the evening hours more system capacity than in the morning hours;

- frequent system users may also experience a relatively low rejection rate.

10. Implementation complexity: An important qualitative criterion for the analysis of congestion control mechanisms is related to the implementation complexity of the mechanism. If the implementation of the mechanism will demand high costs (economical and/or technical) it may not be possible to realise.

\section{PERFORMANCE MODELS AND ANALYSIS}

To emphasise the need for congestion control and scalability, we analysed the performance of a possible UMTS/B-ISDN configuration, using discrete event simulation techniques.

The configuration that we considered consists of a single B-SCP to which one B-SSP is connected as shown in Figure 5. The B-SSP is connected to one RAS and to $n$ FTs. The RAS is connected to $m$ MTs. A Video Server (VS) is connected to the B-SSP, it contains the digital video information (INSIGNIA, 1996).

The RAS, B-SSP and B-SCP entities are modelled as single queue, single server queueing stations. The B-SSP and B-SCP queues are assumed to be finite (50 messages). The RAS queue has infinite length. The VS and the set of FTs and MTs are modelled as infinite server queueing stations.

The scheduling strategies have been selected as follows: The B-SCP and the RAS use FIFO scheduling; the B-SSP uses FIFO scheduling with priority such that call request in progress are assigned a higher priority than new arriving call request.

We have adopted the $\mathrm{B}-\mathrm{VoD}$ information flows defined in the INSIGNIA project (INSIGNIA, 1996). Message processing times have been defined on basis of message length, the values are shown in Table 2 (Bernabei, 1996).

With realistic values for the parameter $\alpha$ the B-SCP is the bottleneck in network performance, that is, the $\mathrm{B}-\mathrm{SCP}$ is the entity that experiences congestion first. 


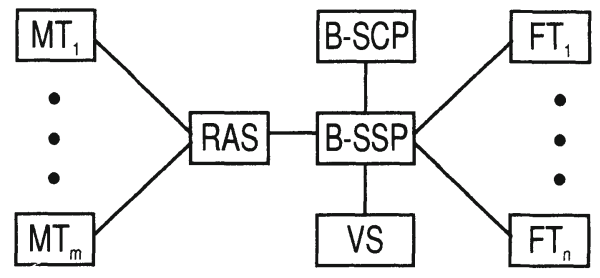

Figure 5 The UMTS/B-ISDN experiment configuration.

Táble 2 Performance model parameter values

\begin{tabular}{lll}
\hline Physical entity & Message(s) & Processing time $(0.1 \mathrm{~ms})$ \\
\hline B-SSP & AddPartyAndBearerToSession & 10 \\
& RequestReportSSMChange & 9 \\
& AddBearerToSession & 8 \\
& JoinPartToSessionAndLinkLegToBearer & 5 \\
& DropParty, PromptAndCollectUserlnformation, & \\
& PlayAnnouncement & 3 \\
& ReleaseSession & 1 \\
\hline B-SCP & ServiceRequest & $14 \alpha$ \\
& ReportSSMChange & $6 \alpha$ \\
& CollectedUserlnformation & $2 \alpha$ \\
& PlayAnnouncement & $\alpha$ \\
\hline RAS / VS / & Setup & 4.5 \\
MT / FT & CallProceeding, Connect & 1.5 \\
& ConnectAcknowledge, Release, ReleaseComplete & 1 \\
\hline
\end{tabular}

Within the simulation model, traffic is generated using a Poisson arrival process. The traffic generated by the fixed users is 6.6 times higher than the traffic generated by the mobile users (MAGIC, 1995). In the simulation experiments, the traffic generated by the mobility procedures such as paging, location updates and handovers have not been included, but will be in further experiments. In the analysis we have focused on network throughput as function of the call request rate.

The scalability experiments that we have performed are based on the procedure described in Section 4 of this paper. We assume the 'desired' throughput as shown in Figure 6. The resource parameter considered in the experiment is the BSCP processing speed (which is indirect proportional to parameter $\alpha$ in Table 2), and as scalability parameter we have selected the number users (which alternatively is reflected in the $\mathrm{B}-\mathrm{VoD}$ call requests rate).

Three successive simulation experiments have been conducted with $\alpha=6, \alpha=2.7$ and $\alpha=1.67$ respectively, the point estimates are shown in Figure 6 . 


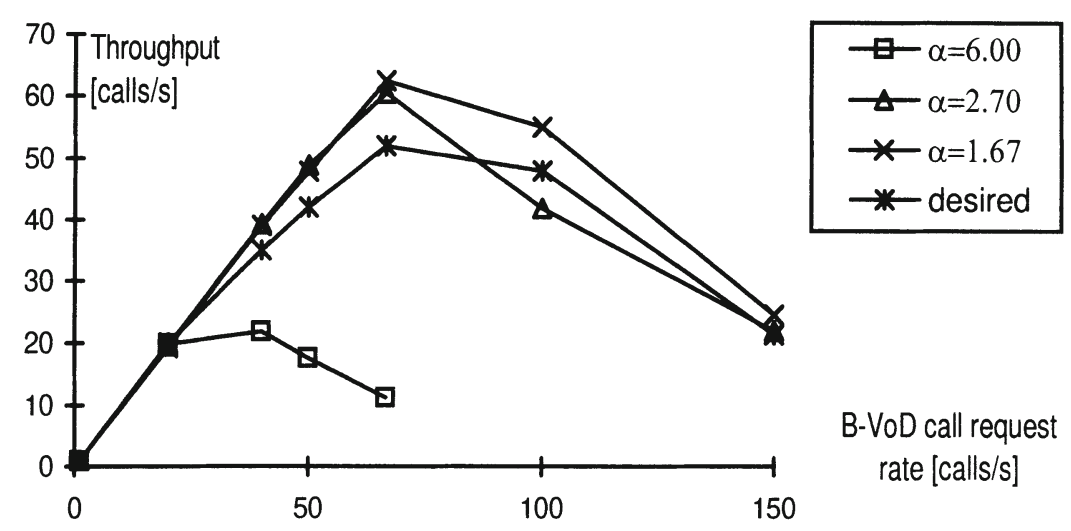

Figure 6 The network throughput as function of the call request rate.

In the analysis the confidence coefficient has been set to 0.99 , the resulting confidence interval at each point is (in the worst case) $\pm 13 \%$ of the point estimate. We can conclude that the 'desired' throughput is satisfied for $\alpha=1.67$.

\section{CONCLUSIONS}

This paper describes some of the critical issues that affect the integration of the UMTS and B-ISDN systems and some solutions are proposed. Furthermore, it addresses a few quantitative (i.e. performance) aspects of an integrated UMTS/BISDN system.

The different UMTS and B-ISDN protocol stacks require interworking functions that extract information from one protocol stack and reformat it for onward transmission via a different protocol stack. Interworking might be defined at different levels in the protocol stack.

Transcoding is an interworking function that changes the source coding format of the user information transported through several links during an end-to-end connection.

During a call, handover is required to transfer the MT connection to each new BTS when the MT roams. From the user's point of view handover should be seamless which implies that a handover should not degrade the QoS (Quality of Service) for the connection as perceived by the user for the support of UMTS/BISDN services, such as B-VoD and B-VC.

For any innovative communication system, scalability of the network is of crucial importance for keeping it commercially interesting. Scalability is the ability to increase the size of a network while maintaining the Quality of Service. The size of a network may refer to several different things, such as: the number of users, the number of network nodes and the geographical spread of the network. 
When the integrated UMTS/B-ISDN architecture has to process an incoming load larger than its capacity then the performance (for example throughput) of the system degrades and eventually the users will experience long delays or a high percentage of service refusals (blocked calls). This state of the system is called congestion. The congestion state can be changed (resolved) by using appropriate congestion mechanisms.

To emphasise the need for congestion control and scalability experiments we investigated the performance of a possible UMTS/B-ISDN configuration by performing simulation experiments.

\section{ACKNOWLEDGEMENTS}

This paper is based on work in the RACE II project MONET (Mobile Networks) and on the ACTS project INSIGNIA (IN and B-ISDN Signalling Integration on ATM platforms) in which the authors participated. The views expressed in this paper are those of the authors and not necessarily those of the projects as a whole. The members of the MONET project, in particular Toon Norp and Marc de Lignie are acknowledged for their help and discussion.

The authors would also like to express their gratitude to Victor Nicola and Phil Chimento for discussions on the subject, and to the European Committee for initiating and sponsoring the RACE and ACTS programmes.

\section{REFERENCES}

ATM Forum (1994) User-network interface (UNI) specification, version 3.1.

Bellcore (1993) AIN SCP generic requirements, GR-1280-CORE, Bellcore, Issue 1. August.

Bernabei, F., Chierchia, G., Gratte, L., and Cuomo, F (1996) Messages weight assignment, internal ACTS INSIGNIA communication.

Broek, J. van der and Katoen, J.-P. (1995) Reference configurations for UMTS, in Proceedings of the 2nd International Workshop on Mobile Multi-Media Communications, A1/1/1-6.

Colombo, G. and Hegeman, H. (1994) Network architecture and functionalities for UMTS, in Proceedings of Wireless Communication Networks (eds. J. Weber, J. Arnbak and R. Prasad), Volume 3, 844-851.

Houck, D.J., Meier-Hellstern, K.S., Saheban, F. and Skoog, R.A. (1994) Failure and congestion propagation through signalling controls, in Proceeding of the 14th International Teletraffic Conference (eds. J. Labetoule and J.W. Roberts), 367-376. 
Hubig, W., and Weber, D. (1994) Overload control in ISDN PABXs, in Proceeding of the 14th International Teletraffic (eds. J. Labetoule and J.W. Roberts), 243-252.

INSIGNIA (1996) First trial: description of the selected services, project document reference number AC068/CLT/111/DS/P/001/b1 (public).

ITU-T I.130, ITU-T Recommendation I.130, .Method for the characterisation of telecommunication services supported by an ISDN and network capabilities of an ISDN.

ITU-T I.350, ITU-T Recommendation I.350, General aspects of service and network performance in digital networks.

ITU-T Q.1214, ITU-T Recommendation Q.1214, Distributed functional plane for Intelligent Network CS-1.

ITU-T Q.2931, ITU-T Q.2931 reference, DSS2 access signalling for B-ISDN.

ITU-T Q.2962, ITU-T Q.2962 reference, DSS2 bandwidth negotiation capability.

ITU-T Q.543, ITU-T recommendation Q.543, Blue Book, Digital exchange performance design.

Karagiannis, G., Katoen, J.-P. and Niemegeers, I.G.M.M (1996) B-ISDN to the cell site switch versus B-ISDN to the mobile terminal, in Proceeding of the IEEE ICCS/ISPACS '96, Singapore, Volume 2, 629-633.

Karagiannis, G., Lignie, M.C. de, Bie, J. de and Niemegeers, I.G.M.M (1997) Handover mechanisms in ATM-based mobile systems, to be published in Journal of Selected Areas in Communications.

Katoen, J.-P. (1995) Functional Integration of UMTS and B-ISDN, in Proceedings of the 45th IEEE Vehicular Technology Conference, Chicago (Illinois), Volume 1, 160-164.

Korner, U., Nyberg, C., and Wallstrom, B. (1994) The impact of new services and new control architectures on overload control, in Proceeding of the 14th International Teletraffic Conference (eds. J. Labetoule and J.W. Roberts), 275-282.

MAGIC (1995) B-ISDN Signalling performance: issues and analysis, document reference R2044/BTL/DP/DS/P/014/b1 (public).

MONET (1995a) Interoperability and integration of UMTS in a B-ISDN backbone, document reference R2066/RMR/UNA2/DS/P/100/b1 (public).

MONET (1995b) Recommendations of UMTS integration scenario's in the BISDN backbone, document reference R2066/RMR/UNA2/DS/P/107/b1 (public).

MONET (1995c) UMTS system structure document, document reference R2066/BT/PM2/DS/P/113/b1 (public).

Norp, T. (1994) Protocol stacks for an integrated UMTS user access network, in Proceeding of the RACE mobile telecommunications workshop, Amsterdam, 310-313.

Pham, X.H. and Betts, R. (1994) Congestion control for intelligent networks, Computer networks and ISDN systems, 26, 511-524. 
Schwartz, M. (1995) Network management and control issues in multimedia wireless networks. IEEE Personal Communications Journal, 2(3), 8-16.

Smith, D.E. (1995) Ensuring robust call throughput and fairness for SCP overload controls. IEEE/ACM Transactions on networking, 3(5), 538-548.

Turner, J.S. (1986) New directions in communications. IEEE Communication. Magazine, 25(10), 8-15.

\section{BIOGRAPHY}

G. Karagiannis received a bachelors degree in Electrical Engineering at the T.E.I Athens, Greece in 1987. In 1988 he joined the laboratory of Network Theory of the Electrical Engineering department at the University of Twente, the Netherlands, where he was involved in VLSI design projects. He received a MSc. degree in electrical engineering at the University of Twente in 1993. In 1995 he graduated for a post graduate designers course at the Tele Informatics and Open Systems (TIOS) group at the University of Twente.

In 1995 he joined the CTIT at the University of Twente. His areas of interest include mobile communication systems, network control mechanisms, performance modelling and analysis. He has actively participated in the RACE-II project MONET and is presently actively involved in the ACTS project INSIGNIA.

B.J.F. van Beijnum was born in Nijmegen, the Netherlands in 1961. In 1985 he received a MSc. degree in electrical engineering and in 1995 he received a Ph.D. degree at the University of Twente. In 1985 he joined the laboratory for network theory of the electrical engineering department at the University of Twente where he worked on formal description and verification methods and their application for VLSI design. In 1994 he joined the Inter University Micro Electronics Centre (IMEC) in Leuven, Belgium.

Since 1995 he is a CTIT staff member at the University of Twente. His present areas of interest include high speed communication network architectures, signalling, performance analysis and performance measurements. He is actively involved in the ACTS project INSIGNIA.

I.G.M.M. Niemegeers was born in Gent, Belgium in 1947. He received a degree in electrical engineering from the Rijksuniversiteit Gent in 1970. In 1972 he received a MSc. E. degree in computer engineering and in 1978 a Ph.D. degree from Purdue University in West Lafayette, Indiana, USA. From 1978 to 1981 he was a designer of packet switching networks at Bell Telephone Mfg. Cy., Antwerp, Belgium.

Since 1981 he is a professor at the Computer Science Department of the University of Twente, Enschede, the Netherlands. He is presently Scientific 
Director of the Centre for Telematics and Information Technology of the University of Twente. His areas of interest are communication systems and performance analysis. He is active in research on integrated networking, highspeed networking, B-ISDN, optical networking, performance analysis and performability. 